\title{
Diseño curricular de un programa académico en turismo, a partir de núcleos problémicos: una experiencia regional
}

Marleny Torres Zamudio ${ }^{1}$

\begin{abstract}
Resumen
Los problemas que aquejan a determinados sectores económicos, identificados a partir de mapas de conocimiento de las regiones, se constituyen en un insumo base para el planteamiento de ofertas académicas de educación superior, bajo el modelo curricular por núcleos problémicos. Este artículo presenta la experiencia de identificación de núcleos problémicos como elementos centrales en el planteamiento de una propuesta de diseño curricular para el área de turismo. Identificando además, las habilidades y competencias necesarias para desempeñarse dentro de esta disciplina.

Palabras clave: competencias, educación superior, modelo curricular, núcleos problémicos, turismo.
\end{abstract}




\title{
Curriculum design of tourism academic program made from a problematical core: A regional experience
}

\begin{abstract}
The problems facing certain economic sectors identified through knowledge maps of different regions represent a basic input for planning of higher education academic programs, under the curriculum model of problematical cores.

This article presents the experience of problematical cores identification as central elements in planning a curriculum proposed for the tourism area. Identifying additionally skills and competencies needed to perform in this discipline.
\end{abstract}

Key words: higher education, tourism, curriculum model, competencies, problematical cores.

Recibido: 7 de febrero de 2013

Aceptado: 29 de mayo de 2013

\section{Introducción}

Para dar inicio a la experiencia de diseño de un Programa Académico se parte de un marco mental bastante amplio y complejo, dada la responsabilidad frente a temas a los que se debe dar respuesta a la hora de ofrecer un programa académico. Así, la pertinencia institucional, la flexibilidad, la necesidad de cumplir con unos lineamientos emanados del Ministerio de Educación Nacional (MEN), las condiciones académicas de pertinencia social y profesional, las competencias profesionales específicas, significan un gran reto para quienes se asignan esta labor; más aún cuando se habla de un campo que por tradición ha tendido a ser un tanto más de carácter práctico y que con frecuencia se ha desarrollado de manera empírica, o por profesionales de otras áreas, o demostrando habilidades técnicas para desempeñarse en él, como es el Turismo. 
Para la generación de una propuesta de Programa Académico que cumpla con las expectativas al interior de la Universidad, del sector empresarial, de las entidades de orden nacional y por supuesto, de los gustos y preferencias los mismos agentes sociales, quienes quieren insertarse con éxito en el campo del turismo, se parte del reconocimiento de necesidades y realidades de la región, estructurando un mapa de conocimiento regional como apuesta investigativa denominada: "Estudio de necesidades de formación orientado a determinar una oferta académica de turismo en Boyacá".

Con un grupo de docentes de la UNAD se indagó la realidad regional del sector turístico, sus problemáticas y los avances en materia de formación académica, presentando los hallazgos detectados en un documento diagnóstico del sector turístico, base para el planteamiento e implementación de procesos formativos en la Universidad. Para reconocer las necesidades del sector turístico fue necesario generar o buscar espacios de interacción con los actores del turismo, empresarios, trabajadores del sector, estudiantes, docentes de otras Universidades y el Sena, representantes del Estado que laboran en función del turismo, entre otros; que finalmente permitirán la dinamización de una propuesta formativa que pueda generar desarrollo en las regiones.

La expedita globalización obliga a las empresas turísticas a buscar la competitividad y calidad, como única manera de sobrevivir en un mundo complejo y desafiante. Se hace necesario entonces la inversión en entrenamiento, formación y desarrollo de las comunidades que atienden servicios turísticos. Respecto a la competitividad:

"Existen diferentes factores que permiten que un destino turístico se considere competitivo, que en principio se encuentran ligados a la adecuación de la oferta de productos y servicios, para satisfacer la demanda, mejorar los mercados y las acciones de promoción. Esta oferta la integran por una parte, el conjunto de atractivos turísticos, los cuales requieren el soporte de infraestructura para la conectividad aérea, terrestre, telecomunicaciones, seguridad y servicios de salud, y de otra parte, la oferta de servicios en los que el capital humano, sus competencias y destrezas, las empresas y los encadenamientos productivos, son el complemento necesario para el fortalecimiento de los destinos" (Bravo y Pérez, 2009:5).

En cuanto a la inversión en entrenamiento y formación Redín y Castilla (citada en Silva y Pensado, 2010) plantean que:

“...un problema del que puede adolecer el sector turístico es la falta de formación especializada en todos los niveles de la empresa. Cada trabajador debería conocer bien su trabajo, para ello la empresa debería tener un programa de formación para los trabajadores novatos, 


\section{Marleny Torres Zamudio}

Diseño curricular de un programa académico en turismo, a partir de núcleos problémicos: una experiencia regional.

planificar sesiones de aprendizaje antes del lanzamiento de nuevos servicios, dar formación sobre la calidad y sus implicaciones y realizar todas las acciones necesarias para que se cumpla el principio expuesto. González y Talón (2003) también coinciden en este aspecto, cuando señalan que la formación es una de las grandes carencias del sector turístico" (p. 2).

\section{Fundamentos Teóricos}

La Universidad Nacional Abierta y a Distancia (UNAD) en los últimos años ha adelantado un proceso de innovación curricular de los programas académicos que quiere ofrecer a la sociedad, partiendo de la aproximación a las necesidades de esa sociedad, núcleos Integradores de Problemas y Núcleos problémicos. Para la Universidad es:

"...de vital importancia para el futuro institucional, el reconocimiento de sus propios elementos y la valoración de los mismos bajo la perspectiva de la adecuación de los fines que orientan el mejoramiento de los procesos formativos, de investigación y desarrollo regional. En este marco de ideas, actúa la innovación académica unadista, la cual requiere un proceso de coconstrucción que evidencie los productos de la inteligencia colectiva y la capacidad de la comunidad académica para repensarse en el marco de las apuestas formativas Unadistas..." (Unad, 2011).

De esta manera, como lo plantean González y Arcila (2006), reconocemos cómo a partir de la instauración generalizada de lo que optamos por denominar currículos problémicos o estructuras curriculares integrales de naturaleza problémica, existiría una gran posibilidad de transfigurar la educación en cuanto a la forma de producción del conocimiento, puesto que nos permitiría pasar de un conocimiento disciplinar, caracterizado por el academicismo, la homogeneidad en las habilidades y la jerarquización, a un conocimiento transdisciplinar, caracterizado por la flexibilidad, la aplicabilidad, la heterogeneidad, la horizontalización de las jerarquías y las posibilidades que ofrece de ser sometido permanentemente al escrutinio público y social (p. 180).

En la UNAD, el diseño curricular está sustentado en la formulación de núcleos problémicos los cuales tienen como fuente las demandas y necesidades presentes en el contexto regional y local, así como en el disciplinar. El diseño curricular por núcleos problémicos implica la formulación de problemas fundantes que representan de un modo estructural preguntas esenciales acerca de una disciplina y contribuyen a su desarrollo y al mejoramiento de la calidad 
de vida de las comunidades. Los núcleos problémicos surgen entonces, de las demandas y necesidades de los contextos locales y regionales y del contexto disciplinar mismo. De ahí su carácter dinámico y problematizador y el hecho de que se constituya en una oportunidad de formación para la vida (UNAD, 2011:91).

Se piensa en un diseño curricular que aporte a la solución de problemáticas del sector turístico, mediante la determinación de núcleos problémicos entendidos como una estrategia curricular de globalización, concreción, especificidad e interdisciplinariedad, que integra la docencia, la investigación y las actividades de extensión que señala problemas concretos teórico práctico y que permite iluminar los procesos académicos de la educación para llegar a la transformación de la realidad (Flórez, Ramírez y Zamora, 2010).

Así también, con la influencia de los enfoques de la teoría sociológica de Durkheim, se hace imprescindible la identificación y análisis de las competencias laborales y profesionales, porque como quiera que se definan o se evalúen, es necesario identificar cuáles posee el individuo y cuáles requiere en su práctica social, económica, cultural o académica, para que pueda ser medido y calificado.

Richard Boyatzis, citado por Companioni (2006) sistematizó los conocimientos de la Psicología en la práctica organizacional cuando refirió que:

"Las competencias son características subyacentes en una persona que están causalmente relacionadas con un desempeño de éxito. Este enfoque teórico está fundamentado en las características psicológicas de los individuos como sustento de una actuación o desempeño laboral exitoso. Numerosos autores se han referido a este polémico tema, y han asumido definiciones que van desde la visión de que éstas no son más que conocimientos, destrezas y aptitudes que se relacionan causalmente con un desempeño exitoso, hasta la valoración de la capacidad productiva de los individuos a partir de experiencias y situaciones concretas del ejercicio profesional" (p. 7).

La Organización para la Cooperación y el Desarrollo Económico (Citado por Pérez, 2009), establece que para que una competencia pueda ser seleccionada como clave o básica, debería cumplir tres condiciones: contribuir a obtener resultados de alto valor personal o social, poder aplicarse a un amplio abanico de contextos y ámbitos relevantes y permitir a las personas que la adquieren superar con éxito exigencias complejas. Es decir, las competencias son básicas o claves cuando resultan valiosas para la totalidad de la población, independientemente del sexo, la condición social y cultural y el entorno familiar (p. 2). 


\section{Marleny Torres Zamudio}

Diseño curricular de un programa académico en turismo, a partir de núcleos problémicos: una experiencia regional.

\section{Metodología}

Para cumplir con los propósitos, se parte entonces de la configuración de un mapa de conocimiento regional, tomando como referencia metodológica los lineamientos dados por la Vicerrectoría de Desarrollo Regional y Proyección Comunitaria de la UNAD, como estrategia para obtener un mayor conocimiento de la región y profundizar en las dinámicas y posibilidades que tiene como territorio, sus problemáticas, y potencialidades en el Sector Turístico. Se inicia con una discusión y reflexión profunda de cuáles son los ámbitos de indagación sobre los que se va a desarrollar.

De esta manera, el mapa se adelanta a través de cuatro fases: una primera que define no solamente los ámbitos de indagación, sino las preguntas problematizadoras para cada ámbito, todo desarrollado a través de un ejercicio colectivo del Cuerpo Académico de la Escuela de Ciencias Administrativas, Contables, Económicas y de Negocios, ECACEN de la UNAD en la Zona Centro de Boyacá.

Se plantea además, en esta fase, el diseño y construcción de un mapa mental, el cual estaría publicado en un portal Web, como medio informativo donde se socializa de manera conjunta los avances del estudio. Surge entonces, de este proceso la configuración de un mapa preliminar.

En la segunda fase, el grupo de investigadores recoge y construye un registro sistemático de documentos que se han realizado sobre el tema y que intentan dar respuesta a las preguntas problematizadoras planteadas en la primera fase; así también, se definen las técnicas de recolección de información y el tratamiento que se dará a las mismas, como resultado de esta fase, se presenta la configuración de la segunda versión del mapa.

Para la tercera fase, se proyectó el desarrollo de foros de regionalización, no sólo en la comunidad académica interna sino en la región, determinando la forma como se visibiliza la región en un futuro cercano y cómo el sector está dispuesto a ser parte de ese futuro. Finalmente, en una cuarta fase, a partir del mapa de conocimiento de la región, se estructura un documento que permitirá, a la Universidad y para la Región, consolidar una propuesta de oferta académica en el área de turismo. 


\section{Resultados y discusión}

\section{Matriz de ámbitos de indagación.}

La Matriz de Ámbitos se desarrolló a partir del estado de arte, que proporcionó el conocimiento del sector y a través de una lluvia de ideas o cuestionamientos informales y grupales sobre posibles situaciones problemáticas, previamente identificadas y priorizadas, se determinaron los ámbitos de indagación a trabajar.

Se generaron las preguntas problematizadoras de indagación por cada ámbito, para luego priorizar situaciones problemáticas, depurándolas de acuerdo con las apuestas productivas y culturales de la región.

Como resultado del trabajo desarrollado por los investigadores, se elaboró una Matriz con los ámbitos: Formación en Turismo, Operadores y Servicios Turísticos, Aporte Económico del Turismo, Infraestructura Turística y Política Sectorial.

\section{La formación en el campo del turismo}

Para establecer un diagnóstico de la formación de talentos humanos en el sector turístico, se analizó la información que reporta el Ministerio de Educación Nacional de Colombia en el Sistema Nacional de Información de la Educación Superior, (SNIES). Se presentan 45 programas aprobados a nivel técnico profesional, 62 programas a nivel tecnológico, 26 programas con Registro Calificado, 2 programas con Acreditación de Alta Calidad en Pregrado, 13 programas de Especialización, y 2 Programas a nivel de Maestría.

La metodología en la que se ofrecen los programas, a nivel técnico solo una institución ofrece el programa a distancia (tradicional) 2 a distancia virtual y 42 de manera presencial. En las tecnologías, 7 a distancia (tradicional), 3 a distancia virtual y 52 presencial. En la metodología de los programas de pregrado hay 6 a distancia (tradicional), 1 distancia virtual y 21 presencial. Para especializaciones 1 a distancia (tradicional), 1 a distancia virtual y 11 presencial. En el caso de las maestrías la metodología utilizada es presencial para los dos programas ofertados.

Se puede observar en la tabla 1 el número promedio de créditos académicos, periodos, y origen de las instituciones que ofrecen programas de formación en el área turística. 


\section{Marleny Torres Zamudio}

Diseño curricular de un programa académico en turismo, a partir de núcleos problémicos: una experiencia regional.

Tabla 1 Consolidado de la oferta Académica por niveles de formación

\begin{tabular}{|c|c|c|c|}
\hline Nivel de formación. & $\begin{array}{c}\text { Promedio de créditos } \\
\text { académicos. }\end{array}$ & $\begin{array}{c}\text { Promedios } \\
\text { de periodos } \\
\text { académicos. }\end{array}$ & $\begin{array}{c}\text { Origen de la } \\
\text { institución. }\end{array}$ \\
\hline Formación técnica. & 67 & 5 & $\begin{array}{r}9 \text { oficial. } \\
36 \text { privada. }\end{array}$ \\
\hline $\begin{array}{c}\text { Formación } \\
\text { técnologica. }\end{array}$ & 103 & 6 & 20 oficial. \\
\hline Pregrado. & 158 & 10 & 42 privada. \\
\hline Especialización. & 25 & 2 & 6 oficial. \\
\hline Maestría. & 56 & 4 & $\begin{array}{r}1 \text { oficial. } \\
12 \text { privada. }\end{array}$ \\
\hline
\end{tabular}

Fuente: La autora, Datos SNIES, 2012.

De acuerdo con el estudio realizado por la Organización Mundial de Turismo (OMT), a través de su Fundación UNWTO, de los programas de formación técnico profesional, tecnológico y profesional, en su gran mayoría definen un perfil ocupacional para el Departamento de Alojamiento; los demás perfiles ocupacionales definen competencias para los otros departamentos del hotel o para la investigación, la docencia y las actividades turísticas.

"En aproximadamente 29 ciudades de Colombia se ofrecen más de 480 programas de educación continuada (Diplomados, cursos de extensión o de actualización) y no más de ocho post grados (Especialista, Magister o Maestría y Doctorado o PHD), con registro calificado, relacionados directamente con la formación en turismo. Cerca del $13 \%$ de estos programas está relacionado con el departamento de alojamiento y administración del alojamiento; el $80 \%$ está relacionado con la operación y administración del departamento de alimentos y bebidas; y cerca del $7 \%$ de los programas relacionado con la operación y organización de eventos" (UNWTO, 2009:86).

El estudio establece que para el caso colombiano, contrario al desarrollo de los procesos de formación en países de Centro América, la formación de talento humano se orienta hacia la hotelería más que al turismo y es preciso tomar en cuenta la estructura empresarial del país, que en su gran mayoría está conformada por micro, pequeñas y empresas familiares, en donde predomina el empirismo en la ocupación para las actividades operativas, contratando para el nivel ejecutivo profesionales formados en otras áreas.

\section{Desarrollos turísticos en la región}

Para los expertos del turismo consultados, el Departamento de Boyacá en este momento es reconocido como un destino turístico religioso, Agroecológico y Arqueológico. Con desarrollos significativos también en el turismo artesanal. 
Así también, al indagar sobre las tendencias en el mediano y largo plazo, como se puede observar en la gráfica 1, se estableció un fuerte potencial de desarrollo del Ecoturismo, el turismo histórico y el cultural para esta región.

Grafica 1 Tendencias de desarrollo turístico

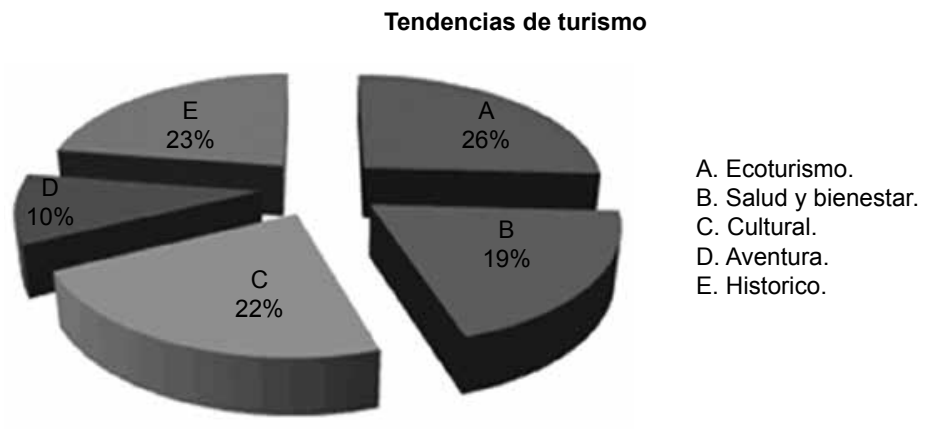

\section{Aspectos Curriculares}

El proceso de definición y selección de competencias exigió un intercambio de ideas con el sector productivo, representantes del gobierno y en general expertos del sector, que ofrecieran las bases para que al interior del grupo de investigadores se diera el intercambio y el debate de ideas; discusiones que llevarían a despejar interrogantes sobre el tipo de turismo que se está desarrollando y se espera potencializar en la región, sobre el profesional que se desea formar, las competencias a desarrollar en ellos y en general características propias de su cultura y sus problemas sociales.

De esta manera, mediante la aplicación del método Delphi, recogiendo apreciaciones de los expertos, a través de sucesivas rondas de cuestionarios, se presentan las competencias más relevantes que requieren los profesionales en el área de turismo.

La oferta académica en turismo ha de tener un componente importante en idiomas y competencias comunicativas con un enfoque cultural, es decir, que permita a los profesionales turísticos no solo comunicarse con sus coterráneos y visitantes, sino ampliar su eje de comunicación, basados en una excelente dialéctica y estilo comunicativo que logre dar a conocer no solamente aspectos culturales propios de la región, sino que además, le permita la interacción a través del conocimiento de otras culturas.

Otro componente que se considera transversal es la utilización de las tecnologías de la información y la comunicación, TIC, como herramienta fundamental de acceso a las nuevas tecnologías que permiten el acercamiento a un mundo más competitivo y dinámico, enfocado a adecuar y facilitar los procesos de calidad del servicio y encaminados a mejorar los estándares que exige el sector. 


\section{Marleny Torres Zamudio}

Diseño curricular de un programa académico en turismo, a partir de núcleos problémicos: una experiencia regional.

Artículo original producto de la investigación.

De igual manera, fortalecer el manejo y los conceptos de mercadeo electrónico desde todas sus dimensiones, como un tema de fundamento competitivo y que se encuentra estrechamente ligado con el desarrollo de las TIC, y el cual es complementado por los criterios de Inteligencia Competitiva y Vigilancia Tecnológica.

La formación en competencias gerenciales le permitirá al profesional del sector turístico contar con unos instrumentos que exige el momento económico productivo del mundo de hoy, como visionario de negocios y generador de estrategias de desarrollo de las comunidades.

En cuanto a las competencias metodológicas, el consenso de expertos como se observa en el gráfico 2 y luego con la discusión entre los académicos al interior de la Universidad, se consideró trascendental para el buen ejercicio profesional, las competencias en servicio al cliente, el manejo ambiental, la organización de eventos, la logística, el manejo de finanzas internacionales y el comercio electrónico.

\section{Grafica 2 Competencias.}

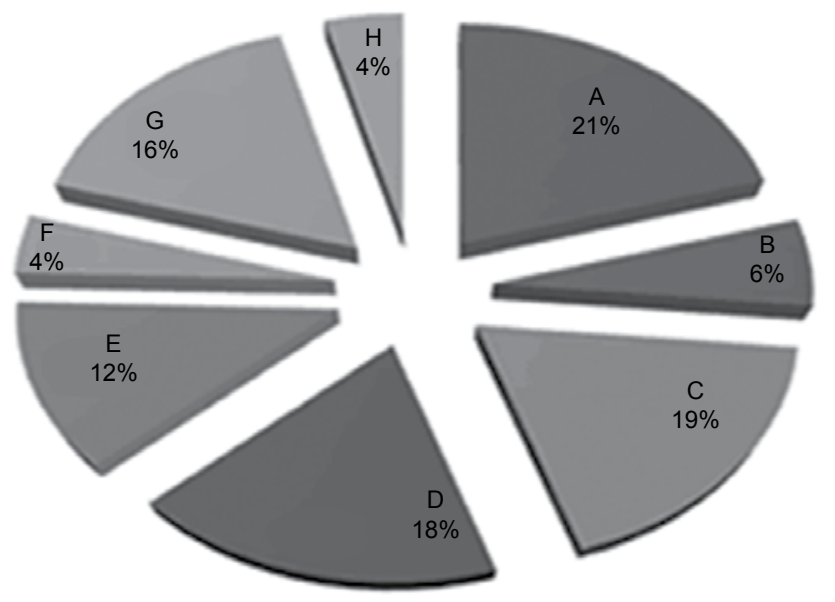

A. Servicio al cliente.

B. Logística.

C. Manejo de TIC

D. Consevación ambiental.

E. Organización de eventos.

F. Manejo de finanzas internacionales.

G. Idiomas.

H. E-Commerce.

De igual manera para los expertos, el trabajo en un contexto internacional, las habilidades en relaciones interpersonales, el reconocimiento a la diversidad y multiculturalidad, la sensibilidad a temas medioambientales tiene una alta favorabilidad. El liderazgo, la capacidad de análisis, síntesis y razonamiento crítico marcan una tendencia importante, el aprendizaje autónomo, la adaptación a nuevas situaciones, la creatividad e iniciativa y la motivación por la calidad, también resultan ser necesarias en la formación de los futuros profesionales del turismo. 


\section{Núcleos problémicos}

\section{-La innovación como estrategia para dinamizar el turismo}

El análisis problemico, debe tener en cuenta las necesidades de formación que el sector requiere en el entorno regional, nacional y mundial y las carencias respecto a esas necesidades en Turismo; estos requerimientos profesionales se han detectado basados en la situación diagnostica arrojada por el mapa de conocimiento regional donde se determina que en el sector el manejo de las Tics y del mercadeo On line, han dado paso a lo que se denomina el e-turismo, que une al turismo y la tecnología necesariamente para el desarrollo de las empresas del sector. Así también, dentro de los procesos de innovación el manejo competente de lenguas extranjeras y el conocimiento de diversos contextos culturales a nivel internacional refleja la competitividad de la empresa turística o servicio que se ofrece. Se da paso entonces, a establecer la "innovación como estrategia para dinamizar el turismo" como necesidad de permanente atención dentro del currículo, como primer núcleo problemico, que hará parte de la estructura de formación profesional integral en esta área.

Algunas de las necesidades específicas que resultaron en el proceso de consulta y debate con los expertos del sector fueron:

*Necesidad de fomentar y desarrollar destinos turísticos mediante el uso masivo de las tecnologías de la información y de las comunicaciones.

*El e- turismo, se constituye en un reto que implica repensar los modelos de gestión y comercialización en las empresas turísticas.

* Exigencias del mercado mediante la gestión de servicios turísticos impone manejar herramientas ofimáticas integradas, conocer y emplear a profundidad tecnologías y soportes informáticos aplicables al sector.

*Necesidad de desarrollar competencias lingüísticas como mínimo en dos idiomas diferentes al materno, que garanticen la comunicación efectiva con turistas.

*La globalización exige el desempeño en medios socioculturales diferentes.

\section{-Gestor de empresas y planificador del turismo con enfoque emprendedor y solidario}

El turismo se ha convertido en un factor de desarrollo en el mundo; es por esta razón que se requiere diseñar programas académicos que se adapten a las tendencias de desarrollo económico desde un ámbito de competitividad y productividad, cumpliendo con los estándares y exigencias del mercado global y permitiendo el fortalecimiento del sector, buscando propiciar altos niveles de desarrollo y bienestar económico. 


\section{Marleny Torres Zamudio}

Diseño curricular de un programa académico en turismo, a partir de núcleos problémicos: una experiencia regional.

Se identificaron necesidades así:

* La generación de empresas que dinamicen el sector.

*Formalización empresarial de negocios que atienden servicios turísticos como hostales, restaurantes, agencias de viaje, operadores turísticos, etc.

*Necesidad de crear proyectos empresariales que generen desarrollo y favorezcan a la región y su comunidad.

* Fomentar la integración empresarial para así aprovechar las ventajas comerciales que ofrece la región. Se evidencia desarticulación, falta de asociatividad y motivación para el trabajo en equipo.

*Desarrollo de productos y actividades turísticas como planes turísticos y excursiones.

*La planificación y gestión pública de destinos turísticos.

*Se requieren líderes que identifiquen necesidades y desarrollen estrategias encaminadas a dinamizar el sector Turístico.

*El sector adolece de planes, programas y políticas que logren posicionar al turismo como un sector que jalona el desarrollo económico y el bienestar social.

\section{-Competitividad y calidad de los servicios del Sector Turístico}

Con respecto a la calidad en la prestación de servicios turísticos, es necesario dar respuesta a los requerimientos de los consumidores y a las demandas del mercado mundial, cumpliendo con estándares de calidad en la prestación de los servicios, a través de procesos de normalización y certificación.

Una de las debilidades que presentan los servicios turísticos ofertados, tiene relación directa con la calidad, es importante para el sector desarrollar estrategias de mejoramiento en áreas como la comodidad, salubridad, seguridad, accesibilidad tecnológica y económica a la infraestructura física, así como a la atención y servicio al cliente.

Algunas de las problemáticas detectadas fueron:

*Deficiencias de calidad en los servicios turísticos ofrecidos.

*Insatisfacción de los turistas y/o visitantes. 
*Falta de conocimiento y capacitación en procesos y de servicios de calidad.

*Carencia de políticas de calidad turística, con la participación de los prestadores de servicios, entes Municipales, Regionales, Nacionales y la comunidad en general.

\section{Conclusiones}

Es preciso mencionar que desde hace ya algún tiempo, previo a la investigación que da origen a este documento, el sector Turismo ha sido de especial interés por parte de un grupo de docentes de la Unad, por lo que ha significado el sector para el desarrollo económico y social en la región. El propósito inicial no era incursionar en este campo de conocimiento con la oferta de programas académicos, sin embargo, al realizar ejercicios de investigación y reconocer el sector, resultó un reto y una gran responsabilidad ingresar en este campo, merecedor de la atención académica e investigativa al interior de la Universidad.

La innovación curricular atada a procesos investigativos permite proponer alternativas de ofertas académicas que surgen de las necesidades del sector y de las comunidades.

El profesional del turismo, debe contar con unas competencias en comunicación asertiva y efectiva, preparase en el dominio de varios idiomas, en el desarrollo de estrategias de marketing basadas en las tics tales como son el e-commerce, e-marketing; diseño de productos y servicios turísticos, planeación y organización y prospectiva del turismo, la vigilancia tecnológica e inteligencia competitiva en sector hotelero y turístico.

El profesional del turismo debe tener profundos conocimientos teóricos y prácticos acerca de la actividad Turística, con amplias capacidades y destrezas gerenciales, habilidad en el manejo de TIC aplicables a la gestión turística y un manejo competente de lenguas extranjeras que le permitan el desarrollo profesional con un enfoque de innovación, solidaridad y competitividad global.

De esta manera, quedan planteadas las bases para la generación de un diseño curricular en el área de turismo, que se gesta en el reconocimiento de realidades regionales, con la participación activa de quienes viven el sector turístico como proyecto de vida y por tanto conocen sus necesidades, así como sus potencialidades. Queda un amplio camino por recorrer, para alcanzar el propósito de presentar un currículo que forme profesionales íntegros que contribuyan al desarrollo de un sector de clase mundial. 


\section{Marleny Torres Zamudio}

Diseño curricular de un programa académico en turismo, a partir de núcleos problémicos: una experiencia regional.

\section{Referencias bibliográficas}

Bravo, A y Pérez, B (2009). Competitividad: el desafio para alcanzar un turismo de clase mundial. Ministerio de Comercio, Industria y Turismo. Obtenido de: https://www. mincomercio.gov.co/minturismo/descargar.php?id=67

Companioni, O (2006). Determinación de las competencias del cocinero profesional para las instalaciones hoteleras de la cadena Islazul, en la ciudad de Camagüey. Universidad de Camagüey. Centro de estudios multidisciplinarios del turismo. Recuperado de: www.repositorio.ehtc.cu/jspui/bitstream/ehtc/26/1/oscar.pdf

Flórez, I, Ramírez, C. y Zamora, H. ( 2010). Portafolio Campo especifico Nivel I Licenciatura en educación básica con énfasis en matemáticas. Recuperado de http:// es.scribd.com/doc/61216318/7/\%C2\%BFQUE-SE-ENTIENDE-POR-NUCLEO-PROBLEMICO.

Flórez, I, Ramírez, C y Zamora, H (2010). Portafolio Campo especifico Nivel I Licenciatura en educación básica con énfasis en matemáticas. Recuperado de http://es.scribd.com/ doc/61216318/7/\%C2\%BFQUE-SE-ENTIENDE-POR-NUCLEO-PROBLEMICO.

González, J. y Arcila, P. (2006). La enseñanza basada en problemas: una estrategia practica de innovación pedagógica. Experiencias significativas en innovación pedagógica. Universidad Nacional de Colombia. Sede Bogotá.

Max-Neef, M. (1993). Desarrollo a escala humana, concepto, aplicaciones y algunas reflexiones. España.

Pérez, C. (2009). Trabajando en el aula con las competencias básicas. Innovación y experiencias educativas. ISSN 1988-6047. Recuperado de http://www.csi-csif.es/ andalucia/modules/mod_ense/revista/pdf/Numero_18/CARLOS_PEREZ_2.pdf

Sánchez, C. (2008) Ministerio de Comercio, Industria y Turismo.. Presentación de la mesa de Mejoramiento de la Competitividad Turística. Encuentro de Turismo Compite, 29 y 30 de septiembre de 2008.

Silva, Y. y Pensado, C. (2010). Evaluación del desempeño y formación en el sector turístico: un estudio bibliometrico desde la psicología de los RRHH. Pasos. Revista de Turismo y Patrimonio cultural ISSN 1695-7121. Vol. 8 N 1 págs. 13-30. 


\section{Revista de}

investigaciones UNAD

Volumen 12. Número 1. Junio 2013

UNAD (2011). I convención Unadista. La academia se pone el overol. Documento sin publicar.

UNAD, (2010) Proyecto Académico Pedagógico Solidario V. 3.0

Varisco, C. (2008). Desarrollo Turístico y desarrollo local: la competitividad de los destinos turísticos de sol y playa. Universidad Nacional de Mar del Plata. Tesis de posgrado - Orientación: economía. Obtenido de http://nulan.mdp.edu.ar/550/1/ varisco_c.pdf 


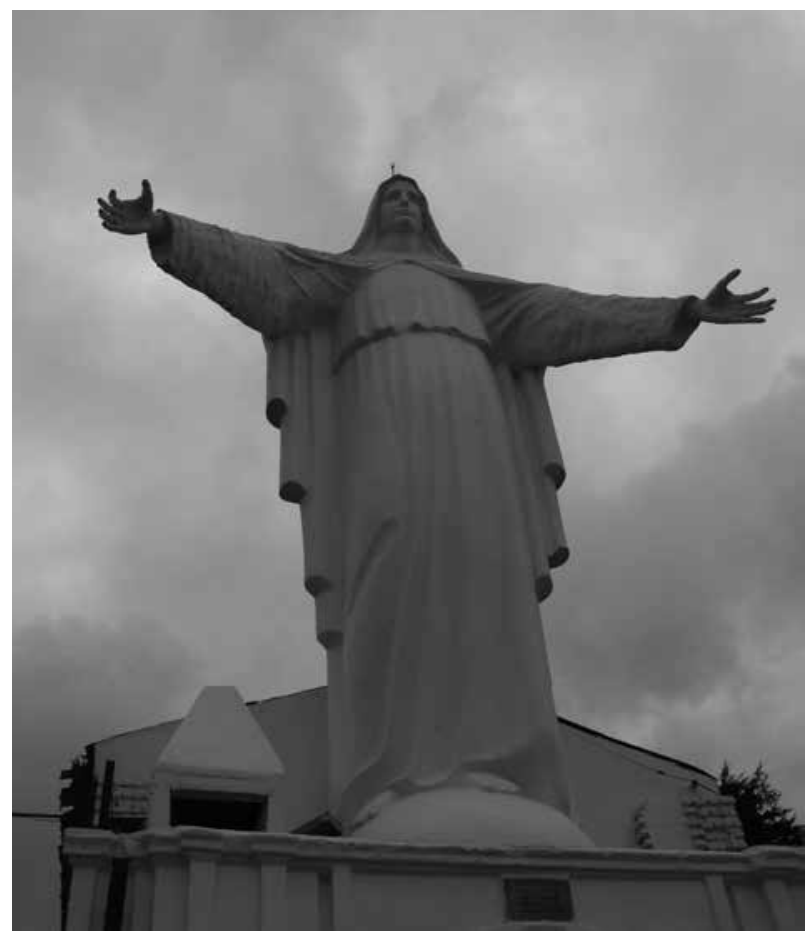

Fotografia: José Enrique Castillo 